\title{
Peritonitis Diverticular: ¿Qué Nos Enseñó Ladies Trial? Análisis Metodológico de su Diseño, Aplicación y Resultados
}

\author{
Diego Hernán Barletta \\ Sanatorio del Oeste (sede Merlo e Ituzaingó). Buenos Aires, Argentina.
}

\begin{abstract}
RESUMEN
Contexto y Antecedentes: LADIES TRIAL es uno de los ensayos más importantes referidos a peritonitis diverticular. A pesar de este y otros ensayos publicados, aún se debate cuáles son los procedimientos adecuados para cada escenario de peritonitis diverticular, haciendo necesaria una revisión profunda de la metodología empleada en los ensayos para validar u objetar sus conclusiones.

Objetivos: Analizar la metodología empleada en el diseño, aplicación, análisis de resultados y conclusiones de sus publicaciones. Secundariamente, colaborar en el mejoramiento de la investigación de la peritonitis diverticular y facilitar el análisis del tema por parte de los lectores.

Métodos: Se analizaron las partes centrales de toda investigación, desde la pregunta de investigación, elaboración de hipótesis, operacionalización de variables y diseño del ensayo, análisis estadístico de resultados y conclusiones. Se buscaron errores, sesgos y debilidades que pudiesen objetar los hallazgos del estudio.

Resultados: LADIES se trató de un estudio randomizado, abierto con análisis de superioridad según intención de tratar modificada en aquellos casos de incumplimiento de los criterios de inclusión y exclusión. Su diseño fue en general correcto, aunque en su aplicación se detectaron errores, debilidades y sesgos. En cuanto a resultados LOLA mostró que en Hinchey III el lavado laparoscópico tiene mayor morbimortalidad temprana que la sigmoidectomía, con un tiempo operatorio menor. Por su parte, DIVA mostró que en Hinchey III y IV la anastomosis primaria tiene mayor sobrevida libre de ostomía con menor morbilidad, combinando la cirugía inicial y cierre ostomía, respecto de la operación de Hartmann.

Conclusiones: El no haber llegado al tamaño de muestra calculado hizo que solo grandes diferencias consiguieran significancia estadística. Las bajas frecuencias de eventos adversos acentuaron este problema metodológico. La especialización de los centros y cirujanos intervinientes, como la exclusión de pacientes hemodinámicamente inestables o bajo corticoterapia comprometieron su validación externa.
\end{abstract}

Palabras clave: Peritonitis Diverticular: Diverticulitis Perforada; Metodología; Investigación; Estudio LADIES

\begin{abstract}
Background: LADIES TRIAL is considered one of the most important trials related to diverticular peritonitis. Its protocol and results were published in 2010, 2015, 2017, and 2019. Despite this one and other published trials, the proper procedures for each diverticular peritonitis scenario are still being debated, a thorough review of the methodology used in this trial is necessary to validate or reject their conclusions.

Aim: To analyze the methodology used in the design, application, analysis of results, and conclusions of all LADIES TRIAL publications. Secondly, to collaborate in the improvement of the research about diverticular peritonitis and to facilitate its analysis by the readers.

Methods: The central parts of a research trial were analyzed, from the research question, hypothesis development, operationalization of variables and trial design, statistical analysis of results, to conclusions. Errors, biases and weaknesses were searched for to try and challenge the trial's findings.

Results: LADIES was a randomized, open-label, superiority trial analyzed according to intention to treat modified in cases of non-compliance with the inclusion-exclusion criteria. Its design was generally correct, although errors, weaknesses, and biases were detected in its application. Regarding results, LOLA showed that, in Hinchey 3, laparoscopic lavage has a higher rate in early morbidity and mortality than sigmoidectomy, but with a shorter operative time. For its part, DIVA showed that, in Hinchey 3 and 4, the primary anastomosis has higher ostomy-free survival with less morbidity, combining the initial surgery and ostomy closure, compared to the Hartmann procedure.

Conclusions: Not having reached the sample size calculated in its design implies that only large effect differences achieved statistical significance. The low frequencies of adverse events accentuated this methodological problem. The specialization of the intervening centers and surgeons, the exclusion of hemodynamically unstable patients or patients undergoing steroid therapy, compromised the external validation of their findings.
\end{abstract}

Keywords: Diverticular Peritonitis; Perforated Diverticulitis; Methodology; Research; LADIES Trial

\section{INTRODUCCIÓN}

El autor no presenta conflicto de intéres.

Diego Hernán Barletta

barlettadie@gmail.com

Recibido: Junio de 2020. Aceptado: Julio de 2020
LADIES TRIAL fue un estudio internacional, multicéntrico que se llevó a cabo desde 2010 a 2016, donde participaron 8 hospitales académicos y 34 hospitales escuelas de Países Bajos, Bélgica e Italia. Su protocolo y resultados fueron publicados en 2012, 2015, 2017 y 2019. ${ }^{1-4}$ Es 
considerado uno de los trabajos más relevantes en peritonitis diverticular. Pese a existir gran cantidad de publicaciones, aún persisten dudas sobre cuáles son los procedimientos más adecuados para cada escenario de peritonitis diverticular.

\section{OBJETIVO}

Analizar desde una perspectiva metodológica su diseño, aplicación y resultados con el objetivo primario de validar o, en su defecto, poner bajo sospecha sus conclusiones. Y con el objetivo secundario colaborar a mejorar la metodología empleada en la investigación del tópico en cuestión y su análisis por parte de los lectores.

\section{METODOLOGÍA}

Se describieron las partes centrales de todo estudio de investigación, por tratarse de un estudio de 2 brazos se analizaron en conjunto las partes que hacen referencia a todo el trabajo y por separado aquellas que son particulares. Se describieron:

- Pregunta de investigación e hipótesis.

- Diseño del estudio.

- Operacionalización de las variables.

- Análisis estadístico y resultados.

- Conclusiones.

En cada una de ellas se buscaron errores, sesgos y debilidades que afectaron la secuencia lógica del proceso de investigación.

\section{RESULTADOS}

\section{Pregunta de Investigación, Hipótesis}

LADIES TRIAL tuvo como objetivo responder a estas dos preguntas: "Primero, ¿Es el lavado laparoscópico (LL) en Hinchey III superior a la sigmoidectomía en términos de morbimortalidad, calidad de vida y costos de salud (brazo LOLA)? Segundo, ¿Cuál abordaje es superior, para Hinchey III o IV, respecto de sobrevida libre de ostomía, calidad de vida y costos de salud, la operación de Hartmann (OH) o la anastomosis primaria (AP) con o sin ostomía de protección (brazo DIVA)?”

En caso de LOLA la hipótesis alterna fue que, para Hinchey III:

- Ha: morbimortalidad a 12 meses de LL < morbimortalidad a 12 meses sigmoidectomía (SG).

- Ha: score de calidad de vida de LL > score de calidad de vida SG.

En caso de DIVA la hipótesis alterna fue que, para Hinchey III o IV:

- Ha: sobrevida libre de ostomía AP (con o sin ostomía)
> sobrevida libre de ostomía $\mathrm{OH}$.

- Ha: score de calidad de vida de AP (con o sin ostomía) $>$ score de calidad de vida $\mathrm{OH}$.

Los análisis de costos no serán abordados debido a las grandes diferencias existentes entre Europa y el ámbito local.

\section{Diseño del Estudio}

Se trató de un estudio randomizado, abierto con análisis de superioridad según intención de tratar, incluyó pacientes entre 18 y 85 años con signos clínicos de peritonitis diverticular con aire libre en $\mathrm{Rx}$ de abdomen o TC con aire libre o líquido difuso. Se excluyeron pacientes con demencia, irradiación pelviana, tratamiento con esteroides, shock o requerimiento de inotrópicos. La randomización fue en bloques de 2, 4 y 6 pacientes, estratificados por edad (mayores y menores de 60 años). En LOLA la distribución fue de 2:1:1 (LL, OH, AP) y de 1:1 (OH, AP) en DIVA. Si bien se detallaron detalles técnicos de los procedimientos, ciertas decisiones quedaron a discrecionalidad del cirujano actuante como el tipo de anastomosis o la decisión de realizar una ileostomía de protección luego de la anastomosis primaria.

\section{Diseño Brazo Lola}

Los grupos se analizaron, como corresponde a todo estudio de superioridad, según intención de tratar, modificada en LOLA solo en 2 casos de violación del protocolo de los criterios de inclusión. La decisión de optar por un "análisis de superioridad" y no realizar un "análisis de no inferioridad” se debió a que este tipo de análisis requiere un tamaño de muestra mayor para obtener un resultado estadísticamente significativo $(\mathrm{p}<0.05)$. El análisis de los pacientes "por intención de tratar" se caracteriza por mantener intacta la randomización de los grupos comparativos y por ser más representativo de la realidad, teniendo en cuenta que al aplicar tratamientos es muy habitual que pacientes asignados a un tratamiento no lo reciban o reciban otro por distintos motivos y/o que haya perdidas en el seguimiento, este tipo de análisis tiene como desventaja la pérdida de homogeneidad de los grupos y por ende pérdida en el efecto esperado del tratamiento evaluado. Existen autores que recomiendan la presentación de los análisis en forma conjunta tanto por intención de tratar como por protocolo, ya que de ser coincidentes en sus resultados le darían mayor robustez al estudio. ${ }^{5-7}$

Los autores esperaban una morbimortalidad del 10\% para el grupo LL y una del 25\% para el grupo SG (operación de Hartmann $(\mathrm{OH})$ y anastomosis primaria (AP), por tanto, la diferencia esperada fue del $15 \%$ y en base a esta diferencia se calculó el tamaño muestral.

De una población de 563 pacientes con diverticulitis per- 
forada 377 fueron apartados por criterio de inclusión y exclusión, quedando 186 pacientes a los que se les realizó laparoscopia diagnóstica, 77 de ellos eran Hinchey I o II y no fueron enrolados, 19 eran Hinchey IV y se enrolaron en DIVA, los restantes 90 pacientes fueron incluidos en este estudio como Hinchey III. Las características de los pacientes excluidos no fueron significativamente distintas de los incluidos. Los grupos se conformaron con $47 \mathrm{pa}-$ cientes en el grupo de LL (45LL, $1 \mathrm{OH}, 1$ exclusión y 1 pérdida de seguimiento) y 43 en el grupo de $\mathrm{SG}(21 \mathrm{OH}$, 20 AP, 1 LL y 1 exclusión). Luego de las exclusiones quedaron 46 en grupo LL y 42 en grupo SG según intención de tratar modificada.

\section{Operacionalizacion de las Variables Brazo Lola}

La variable de resultado primario elegida fue una variable categórica expresada en porcentajes que combina la mortalidad y morbilidad mayor a 12 meses de la cirugía inicial expresada como:

\section{ptes fallecidos o con morbilidad mayor ptes tratados}

Medir morbimortalidad tiene como ventaja aumentar la proporción del evento esperado y por ende su diferencia, permitiendo disminuir el tamaño muestral necesario para obtener significancia estadística.

La morbilidad mayor se definió como reintervención, evisceración, absceso abdominal que requiere drenaje percutáneo, infarto agudo de miocardio, urosepsis, falla renal y falla respiratoria. La morbimortalidad temprana se definió como aquella que ocurrió dentro de los 30 días siguientes a la cirugía o hasta el alta si la internación fue mayor a ese plazo. Se consideró falla de tratamiento a la presencia de sepsis persistente que genere muerte o necesite reoperación. Las sigmoidectomías electivas en el grupo de LL se computaron como reintervenciones.

Las variables resultado accesorias fueron el tiempo operatorio, la estadía, días de vida fuera del hospital, morbimortalidad tardía, eventración, reintervenciones tardías y la variable de resultado secundaria fue la calidad de vida medida por 3 cuestionarios (SF-36, GIQLI y EQ5D).

\section{Análisis Estadístico y Resultados Brazo Lola}

Los test estadísticos empleados fueron los habituales y los datos fueron reportados con medidas de efecto, diferencias de media, odds ratio e intervalos de confianza del $95 \%$.

73/88 (83\%) de los pacientes fueron operados por cirujanos especializados en cirugía digestiva no siendo significativa la diferencia de esta variable dentro de los grupos (37/46 en LL y 36/42 en SG). La validación externa de un estudio hace referencia a la aplicabilidad de los hallazgos de la muestra en la población, debido a que la gran mayoría de los pacientes con peritonitis diverticular son operados por cirujanos generales los resultados del estudio tendrán un sesgo importante en cuanto a la aplicabilidad de sus hallazgos en la práctica diaria.

También se encontraron diferencias (no estadísticamente significativas) tanto en la proporción de pacientes ASA 3 y 4 como en valores de la escala POSSUM-OS, en ambos casos favorecieron al grupo LL. El término estadísticamente significativo solo hace referencia a la $\mathrm{p}<0.05$, es la chance de que las diferencias encontradas en los grupos de estudio se deban al azar. En cambio, clínicamente significativo hace referencia a que tiene importancia o relevancia en la práctica médica.

El estudio fue detenido en febrero de 2013 durante el tercer análisis protocolar de los datos realizado por el comité de seguridad en virtud de la alta tasa de complicaciones tempranas observada en el grupo de LL. Durante los 12 meses de seguimiento no se observó diferencia significativa en morbimortalidad ( 30 pacientes en LL vs 25 pacientes en SG; OR 1.28, 95\% CI 0.54-3.03, p=0.5804). E1 tiempo operatorio medio fue menor para el grupo LL 60 min vs. $120 \mathrm{~min}$ para $\mathrm{SG}$ (diferencia media $-54.53 \mathrm{~min}$, 95\% BCa CI -68.04 a -40.26, $\mathrm{p}=0 \cdot 0010$ ).

Se observó una contradicción en la presentación de los datos de las reintervenciones tempranas. Primeramente, menciona que la morbimortalidad temprana fue mayor en el grupo LL (18 [39\%] pacientes en LL vs 8 [19\%] en SG [OR 2.74, 95\% CI 1.03-7.27, p=0.0427]), lo cual, dicen, puede explicarse debido al alto índice de reintervenciones en LL vs SG (16 y 3 pacientes, OR 6.3, 95\% CI 1.85-26, $\mathrm{p}=0.0041$ ). Sin embargo, luego, el mismo trabajo menciona que la sepsis fue controlada satisfactoriamente, esto es paciente vivo y sin necesidad de reintervenciones, en 35 pacientes de LL y en 38 de SG. Además, menciona que en el grupo LL 9 pacientes con sepsis persistente requirieron reintervenciones y 2 más fallecieron por falla multiorgánica. De igual modo podemos observar que el grupo LL contabilizó 2 muertes, 9 reintervenciones y 9 drenajes percutáneos. Por tanto, no queda claro si realmente se reoperaron 16 pacientes o si se contabilizaron eventos como pacientes, en cuyo caso se incurrió en un error metodológico ya que la variable cantidad de eventos se mide como el promedio de eventos por paciente (cantidad de eventos totales /cantidad de ptes.) y la magnitud del efecto se puede expresar por ejemplo como d de Cohen, pero nunca como OR. ${ }^{8}$

La mortalidad temprana fue $2 / 46(4,3 \%)$ para el grupo LL y $1 / 42(2,4 \%)$ para el grupo SG, mientras que la mortalidad a los 12 meses fue de 4/46 (8,7\%) para el grupo LL y 6/42 (14,3\%) para el grupo SG. 
No se identificaron diferencias significativas en los resultados de los cuestionarios de calidad de vida (SF-36, GIQLI y EQ5D).

Los cierres de las ostomías se realizaron en 5/11 pacientes en el grupo LL y en 24/35 en el SG. El LL fue exitoso en 24/46 (52\%) pacientes entendiéndose que ninguno de ellos requirió otro tratamiento durante la internación como tampoco sigmoidectomía electiva hasta los 12 meses. 36 (78\%) pacientes en el grupo LL y 30 (71\%) en el grupo SG estuvieron vivos y libres de ostomías luego de los 12 meses de seguimiento (OR 1.53, 95\% CI 0.55-4.30, $\mathrm{p}=0.4193$ ). se menciona que dentro de los 88 pacientes enrolados se reportaron 7 (8\%) casos de carcinoma sigmoideo, un tercio de las sigmoidectomías electivas se debieron a ello.

\section{Conclusiones Brazo Lola}

Los resultados del trabajo no permitieron rechazar las hipótesis nulas.

HO: morbimortalidad a 12 meses de $\mathrm{LL}>\mathrm{o}=$ morbimortalidad a 12 meses sigmoidectomía (SG).

HO: score de calidad de vida de $\mathrm{LL}<\mathrm{o}=$ score de calidad de vida SG.

La morbimortalidad temprana luego de la cirugía inicial fue mayor en el grupo LL vs el grupo SG.

El tiempo operatorio fue estadísticamente menor en el grupo LL vs el grupo SG.

Los autores hicieron hincapié en que si bien hubo en LL mayor morbilidad temprana esta no se vio reflejada en una mayor mortalidad y esto podría sugerir que los pacientes que fallarían con el LL podrían ser rescatados sin comprometer su sobrevida. Estas apreciaciones no se desprenden del trabajo ya que la mortalidad no fue evaluada en forma aislada, por ende, diferencias clínicamente significativas en mortalidad podrían no haber alcanzado significancia estadística debido al tamaño muestral empleado.

Según los autores las fallas para controlar la sepsis en el grupo LL podrían atribuirse a un error diagnóstico al confundir peritonitis fecales por purulentas, de hecho, en el grupo SG un tercio de las piezas operatorias tenían perforaciones. Resulta evidente que la sola diferenciación en virtud de la clasificación subjetiva en Hinchey III o IV, no alcanza para identificar aquellos pacientes con perforaciones colónicas persistentes en el intraoperatorio y podría tener un mayor impacto en LL en comparación a las técnicas resectivas, sin embargo, en virtud del diseño del estudio no es posible saberlo. Por tanto, de considerarse esta variable como predictora de resultados relevantes, se deberán incorporar en el diseño de los estudios acciones, reproducibles en la práctica cotidiana, para su correcta medición.

\section{Diseño Brazo Diva}

Luego de terminado tempranamente el brazo LOLA se calculó el tamaño de la muestra según una diferencia de $22 \%$ en la reconstrucción (72-50\%), siendo necesario 212 pacientes para establecer esta diferencia como estadísticamente significativa en un análisis con 2 colas con un $a=0.05$ y un poder de $90 \%(\beta=0.1)$. A los 212 pacientes se les suma un $10 \%$ por posibles pérdidas en el seguimiento, quedando una muestra de 236 pacientes. Al igual que su otro brazo, DIVA terminó prematuramente por dificultades en el reclutamiento de los pacientes. $\alpha$ y $\beta$ son conocidos como los errores tipo 1 y 2 respectivamente, representan la posibilidad de tomar una decisión incorrecta respecto de la hipótesis nula. Error tipo 1 rechaza una hipótesis nula siendo esta verdadera (encuentro un efecto donde no lo hay, $5 \%$ de que esta diferencia sea por azar). Error tipo 2 no rechaza una hipótesis nula siendo esta falsa (no soy capaz de encontrar el efecto donde si lo hay, "no encuentro diferencia entre los grupos, pero me puedo estar equivocando un $10 \%$ de las veces").

El reclutamiento de los pacientes se llevó a cabo de la siguiente manera: En Hinchey III se enrolaron 93 pacientes, 47 en para el grupo $\mathrm{OH}$ ( 1 excluido, $1 \mathrm{AP}, 45 \mathrm{OH}$; de ellos 1 no ostomía, 34 cierres y 11 no cerrados) y 46 en el grupo AP (1 LL, $5 \mathrm{OH}, 40 \mathrm{AP}$; de ellos 13 no ostomía, 29 cerrados y 4 no cerrados). Quedando para análisis según intención de tratar 92 pacientes y para análisis de reconstrucción del tránsito 62 pacientes. En Hinchey IV se enrolaron 40 pacientes, 21 en para el grupo $\mathrm{OH}$ (1 excluido, $20 \mathrm{OH}$; de ellos, 10 cierres y 10 no cerrados) y 19 en el grupo AP (1 excluido, $2 \mathrm{OH}, 16 \mathrm{AP}$; de ellos 1 se perdió, 4 no ostomía, 9 cerrados y 4 no cerrados). Quedando para análisis según intención de tratar 38 ptes y para análisis de reconstrucción del tránsito 19 pacientes.

Según intensión de tratar estos 130 pacientes se dividieron 66 para el grupo $\mathrm{OH}$ y 64 para el grupo AP. 65/66 (98\%) pacientes del grupo $\mathrm{OH}$ recibieron el procedimiento planeado por protocolo mientras que solo 56/64 $(87,5 \%)$ pacientes lo hicieron en el grupo AP. Como se explicó anteriormente el efecto o resultados de la AP quedó diluido, si bien esto no invalida el estudio hay que tener en cuenta esta diferencia al momento de elaborar las conclusiones.

\section{Operacionalización de las Variables Brazo Diva}

La variable de resultado primaria fue la sobrevida libre de ostomía medida 12 meses después de la primera cirugía. Se trata de una variable categórica y temporal.

Existe una discrepancia entre las variables secundarias descriptas en el protocolo de 2010 y la publicación de 2017, en esta última se mencionan como variables de resultado secundario a la mortalidad y morbilidad temprana, a las características de los cuidados preoperatorios y operatorios recibidos y por último hace referencia a la ca- 
lidad de vida. No es el hallazgo de diferencias estadísticamente significativas de las variables entre los grupos comparados lo que da valor a un estudio sino la secuencia lógica que se desprende de la pregunta de investigación, la elaboración correcta de las hipótesis y variables que miden aquello que deseo saber, la correcta selección de la población y muestra, etc. Si todo ello no ocurre solo podré decir que las diferencias halladas son estadísticamente significativas con $\mathrm{p}<0.05$.

\section{Análisis Estadístico y Resultados Brazo Diva}

Los test estadísticos empleados fueron los habituales (Kaplan-Meier, Mantel-Cox y Hazard ratio para la sobrevida, test exacto de Fisher para variables categóricas con bajas frecuencias y test de Student para variables continuas). No hubo diferencias estadísticas entre las características de los grupos. Al igual que en LOLA, 115/130 (88,5\%) de los pacientes de DIVA fue operado por un cirujano especializado en cirugía digestiva, comprometiendo la validez externa de los resultados.

En el grupo OH 65/66 (98\%) pacientes quedaron con ostomía luego del procedimiento inicial, mientras que en el grupo AP solo lo hicieron 46/64 (73\%) pacientes. Se reconstruyeron 44/65 (68\%) pacientes en el grupo $\mathrm{OH}$ vs. $38 / 46$ (83\%) en el grupo AP ( $p=0.085)$. Quedaron excluidos del análisis de reconstrucción del tránsito aquellos pacientes a los que se no se les efectuó una ostomía o que no fueron reconstruidos. Comparar los porcentajes de reconstrucción de los pacientes con ostomía dentro de cada grupo cuando no es la variable de resultado principal del estudio y presentarla junto con una $p>0.05$ aunque estadísticamente correcto puede llevar a confusiones, ver más adelante.

Como principal resultado, los pacientes del grupo AP tuvieron una sobrevida libre de ostomía estadísticamente significativa mayor que los del grupo $\mathrm{OH}(94 \cdot 6 \%$ [95\% CI $88 \cdot 7-100]$ vs $71 \cdot 7 \%$ [95\% CI $60 \cdot 1-83 \cdot 3]$, hazard ratio [HR] 2.79 [95\% CI 1.86-4.18]; test log-rank p<0.0001. HR es la manera correcta de medir en forma dinámica las curvas de sobrevida debido a que no solo me dice si el evento ocurrió o no, sino el tiempo en que tarda el evento en ocurrir. En este caso se interpretaría que los pacientes no reconstruidos del grupo AP tenían en promedio 2,79 veces más chances de reconstruirse en el siguiente intervalo de tiempo que los pacientes del grupo $\mathrm{OH}$. 9,10

No se encontraron diferencias estadísticamente significativas en resultados postoperatorios tempranos del procedimiento inicial. Veintinueve (44\%) de 66 pacientes en el grupo $\mathrm{OH}$ y 25 (39\%) de 64 pacientes en el grupo AP, tuvieron morbilidades mayores o menores. La morbilidad mayor se observó en 8 (12\%) de 66 pacientes en el grupo $\mathrm{OH}$ y en 9 (14\%) de 64 pacientes en el grupo AP. La mortalidad no fue estadísticamente diferente entre los pacientes asignados en ambos grupos (grupo OH 3/66 [3\%] vs. grupo AP 4/63 [6\%]; p=0·44). En cuanto a la morbilidad asociada a la reconstrucción del tránsito, esta fue estadísticamente menor en el grupo AP vs. grupo $\mathrm{OH}(3 / 38$ [8\%] vs. $13 / 66$ [30\%] p=0 023). Si bien no alcanzó significancia estadística, la morbilidad global para el procedimiento inicial y la subsecuente reconstrucción fue menor para el grupo AP vs. grupo $\mathrm{OH}(25 / 63$ [40\%] vs. 37/66 [56\%]; $\mathrm{p}=0 \cdot 078$. Recordar que el efecto esperado del grupo AP esta diluido por el análisis según intensión de tratar. No se encontraron diferencias estadísticamente significativas en los resultados de los cuestionarios de escala de vida en ambos grupos.

\section{Conclusiones Brazo Diva}

Los resultados del trabajo permitieron rechazar la hipótesis nula principal. Se acepta entonces:

Ha: sobrevida libre de ostomía AP (con o sin ostomía) > sobrevida libre de ostomía $\mathrm{OH}$.

Pero no permitieron rechazar la hipótesis nula secundaria, se acepta entonces:

HO: score de calidad de vida de AP (con o sin ostomía) $<\mathrm{o}=$ score de calidad de vida $\mathrm{OH}$.

Se observó además que AP tuvo, respecto de grupo $\mathrm{OH}$, con significancia estadística:

1. Menor morbilidad global temprana luego de reconstrucción del tránsito.

2. Menor tiempo medio para la reconstrucción.

3. Menor estadía postoperatoria luego de la reconstrucción.

\section{DISCUSIÓNY CONCLUSIONES}

Este trabajo representa los resultados de una población europea de primer mundo con peritonitis purulenta o fecal, hemodinámicamente estable, atendida por cirujanos especializados en cirugía digestiva dentro de instituciones de tercer nivel. Cuyos dos brazos fueron interrumpidos tempranamente debido a la elevada tasa de complicaciones tempranas en el grupo LL y por caída en el reclutamiento de pacientes en el brazo DIVA. LADIES tuvo una muestra tan pequeña que solo las diferencias grandes pudieron llegar a la significancia estadística. Es por ello que las bajas frecuencias de eventos adversos relacionadas con la exclusión de pacientes inestables o en tratamiento con corticoides, como también la especialización de los cirujanos y las instituciones que brindaron los cuidados no hicieron más que agrandar este inconveniente y comprometer seriamente su validez externa. Como conclusión LOLA no solo no pudo demostrar la menor morbimortalidad a 12 meses de LL, sino que por el contra- 
rio evidenció una mayor morbilidad temprana de LL que alcanzó significancia estadística. LOLA Tampoco pudo demostrar mejor calidad de vida en LL vs. SG. El análisis de las piezas operatorias detectó en alrededor de un tercio de ellas perforaciones colónicas inadvertidas en el intraoperatorio, hecho que pone en evidencia la subjetividad de la clasificación de Hinchey y la necesidad de objetivar en el intraoperatorio la existencia o no de una solución de continuidad para la correcta estratificación de los pacientes. Por su parte DIVA demostró una mayor sobrevida libre de ostomía tanto a favor de AP vs. OH. También demostró menor morbilidad luego del cierre de AP vs. $\mathrm{OH}$ con significancia estadística. DIVA no demostró diferencias estadísticamente significativas en morbimortalidad ni en calidad de vida entre los grupos.

\section{BIBLIOGRAFÍA}

1. Swank HA, et al.; Dutch Diverticular Disease (3D) Collaborative Study Group. The ladies trial: laparoscopic peritoneal lavage or resection for purulent peritonitis and Hartmann's procedure or resection with primary anastomosis for purulent or faecal peritonitis in perforated diverticulitis (NTR2037). BMC Surg. 2010; 10:29.

2. Vennix S, et al. Laparoscopic peritoneal lavage or sigmoidectomy for perforated diverticulitis with purulent peritonitis: a multicentre, parallel-group, randomised, open-label trial. Lancet. 2015; 386:1269-1277.

3. Vennix S, van Dieren S, Opmeer BC, Lange JF, Bemelman WA. Cost analysis of laparoscopic lavage compared with sigmoid resection for perforated diverticulitis in the Ladies trial. Br J Surg. 2017; 104(1):62-68.

4. Lambrichts DPV, et al. Hartmann's procedure versus sigmoidectomy with primary anastomosis for perforated diverticulitis with purulent or faecal peritonitis (LADIES): a multicentre, parallel-group, randomised, open-label, superiority trial. Lancet Gastroenterol Hepatol. 2019; 4(8):599-610.
5. Gupta SK. Intention-to-treat concept: A review. Perspect Clin Res. 2011; 2(3):109-12.

6. Montedori A, Bonacini MI, Casazza G, Luchetta ML, Duca P, Cozzolino F, Abraha I. Modified versus standard intention-totreat reporting: are there differences in methodological quality, sponsorship, and findings in randomized trials? A cross-sectional study. Trials. 2011; 12:58.

7. Lesaffre E. Superiority, equivalence, and non-inferiority trials. Bull NYU Hosp Jt Dis. 2008; 66(2):150-4.

8. Kim HY. Statistical notes for clinical researchers: effect size. Restor Dent Endod. 2015; 40(4):328-31.

9. Martínez-González MA, Alonso A, López Fidalgo J. ¿Qué es una hazard ratio? Nociones de análisis de supervivencia [What is hazard ratio? Concepts in survival analysis]. Med Clin (Barc). 2008; 131(2):65-72.

10. Molina Arias, M. «Hazard ratio: cuando el riesgo varía a lo largo del tiempo». Pediatría Atención Primaria 2015;17(66): 185-88. 